\title{
Infrared properties of cuprates in the pseudogap state: A study of Mitrović-Fiorucci and Sharapov-Carbotte scattering rates
}

\author{
Pankaj Bhalla* and Navinder Singh \\ Physical Research Laboratory, Navrangpura, Ahmedabad-380009 India.
}

(Dated: August 31, 2018)

\begin{abstract}
The frequency dependent scattering rate of generalized Drude model contains important information on the electronic structure and on scattering mechanism. In the present investigation, we study the frequency dependent scattering rate of cuprates (Mitrović-Fiorucci/ Sharapov-Carbotte scattering rate) in the pseudogap phase using the non-constant energy dependent Yang-Rice-Zhang (YRZ) density of states. First, with the energy dependent density of states, the scattering rate shows a depression at low energy coming from the opening of the pseudogap. Second, the evolution of $1 / \tau(\omega, T)$ with temperature shows the observed increase in scattering rate with temperature at lower frequencies and the temperature independence of $1 / \tau(\omega)$ at higher frequencies. Third, the signature of the thresholds due to the boson density of states and to the electronic density of states are also observed. These signatures are qualitatively in accord with the experiments.
\end{abstract}

PACS numbers: $74.25 \mathrm{Gz}, 74.72-\mathrm{h}, 72.10 \mathrm{Di}$

\section{INTRODUCTION}

High Temperature Superconductors such as cuprates 1 are strongly correlated systems ${ }^{2}$ having electron-electron interaction energy much greater than the electronic kinetic energy on the low doping side of the phase diagram and the opposite on the high doping side. In the intermediate regime of optimal doping both are of comparable magnitude. This interplay of strong electron-electron interaction energy with their kinetic energy leads to the interesting phases as a function of temperature and doping. A typical phase diagram of the cuprates is shown in Fig. 1(a), It has three different phases, antiferromagnetic (AF) phase, superconducting (SC) phase and the pseudogap (PG) phase. The present investigation deals with the pseudogap state, where physical properties of cuprates show anomalous behavior. These features are observed at the temperature $T<T^{*}$, where $T^{*}$ is the temperature below which the pseudogap is observed. Several attempts have been made to explain the physics of this region, but still there are some open questions, for example, the microscopic origin of the pseudogap state? What is the relationship (if any) of the pseudogap and superconducting gap? etc. The pseudogap is seen in many different experimental probes like Nuclear Magnetic Resonance (NMR), knight shift, neutron scattering, Angle Resolved Photoemission Spectroscopy (ARPES), tunneling experiments, etc. A clear manifestation of the pseudogap is also seen in infrared spectroscopy. In infrared spectroscopy, the reflectance of a given sample (at a given temperature and doping) is measured and then by Kramers-Kronig (KK) transformations, the infrared/optical conductivity $\sigma(\omega)$ is calculated. Below a temperature $T^{*}$, a depression is observed in the mid-infrared region of the conductivity which is a signature of the pseudogap formation ${ }^{3}$ as shown schematically in Fig. 1(b) and 1(c). A more direct manifestation is observed in the frequency dependent scattering rate $1 / \tau(\omega, T)$ of Generalized Drude Model (GDM) calculated from the experimental conductivity.
This manifestation occurs in the form of a depression below some characteristic frequency in $1 / \tau(\omega, T)$. The representation of conductivity through frequency dependent scattering rate and mass enhancement factor is based on the work of Mori and Allen ${ }^{415}$, which is a generalization of the standard Drude model, when the electron-phonon coupling is important. The GDM can also be derived from Langevin equation with time dependent friction and it is of general validity ${ }^{6}$. Allen's model gives a simple expression for the scattering rate in terms of the electronphonon spectral function at zero temperature ${ }^{5}$. This is further extended for finite temperature by Shulga, Dolgov and Maksimov ${ }^{7}$. But both of these formalisms are under the assumption of the constant Electronic Density Of States (EDOS) at the Fermi energy. Mitrovic and Fiorucci 8 have given a relation for non-constant EDOS at zero temperature that has been generalized recently by Sharapov and Carbotte ${ }^{9}$ for finite temperatures.

Much work ${ }^{10 / 11}$ has been done to explain the behaviour of $1 / \tau(\omega, T)$ and the presence of pseudogap in cuprates. Puchkov et. al 11 showed that when temperature $T$ is less than $T^{*}$ (the pseudogap temperature), absorption decreases at infrared frequencies which is a signature of pseudogap formation. Due to this decreased absorption, the in-plane conductivity shows the transfer of spectral weight from the low frequency Drude part to the midinfrared part (see Fig. $1(\mathrm{~b})$ and $1(\mathrm{c})$. Timusk et. al $\stackrel{3}{6}$ also explained these experimental results and showed that at low frequencies and at temperature below $T^{*}$, the amplitude of the scattering rate is suppressed more and the mass enhancement factor increases. But no such type of signature has been observed at higher frequencies. The analysis of these results showed that there are two thresholds ${ }^{3}$ occurring in the generalized Drude scattering rate at around $600 \mathrm{~cm}^{-1}$ and $1000 \mathrm{~cm}^{-1}$. The main experimental features of $1 / \tau(\omega, T)$ can be summed as:

1. Depression formation at lower frequencies due to the opening of the pseudogap (refer Fig. 3(a). 


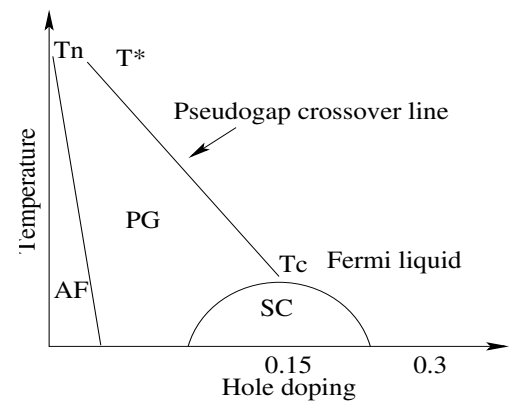

(a)

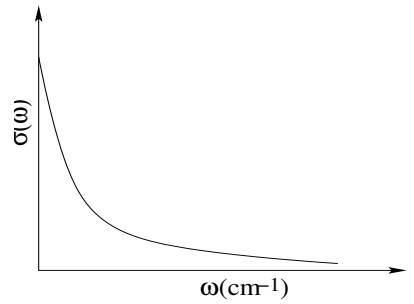

(b)

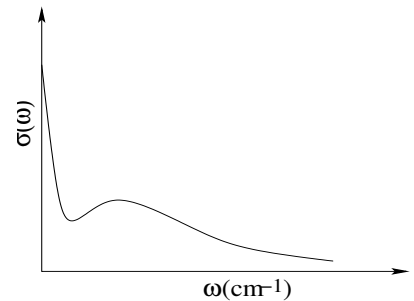

(c)
FIG. 1. (a) The phase diagram of cuprates showing the three different regions with the hole doping and temperature variation. The used symbols are antiferromagnetic (AF), superconducting (SC), pseudogap (PG), Neel's temperature $\left(T_{n}\right)$, Critical temperature $\left(T_{c}\right)$, (b) the variation of the conductivity at temperature $T>T^{*}$ where there is no pseudogap and (c) corresponds to the behaviour of conductivity at $T<T^{*}$ where there is a pseudogap. There is a Drude part at lower frequencies, but there is small hump in conductivity at infrared part which gives the signature of the presence of the pseudogap.

2. Two threshold scenario of $1 / \tau(\omega, T)$ (refer Fig 3(b)].

3. Temperature dependence of $1 / \tau(\omega, T)$ at low frequencies and temperature independence at high frequencies (refer Fig $3(\mathrm{c})$ ).

In the present investigation, we show that the above experimental features of $1 / \tau(\omega, T)$ can be understood using the Mitrovic-Fiorucci/Sharapov-Carbotte formulation of the scattering rate by taking into account the pseudogap formation in electronic density of states. For the pseudogap EDOS, we use the YRZ phenomenological mode ${ }^{12 \mid 13}$ and show that it describes the above mentioned experimental features of $1 / \tau(\omega, T)$ very well. Thus, we report another success of the YRZ model.

The paper has been organized as follows. With the introductory background as given above in section 1, we discuss the theoretical approaches to formulate the scattering rate and the YRZ ansatz in section 2. The numerical results and its analysis are given in section 3 . In the last section, discussion and conclusion are presented.

\section{THEORETICAL FORMALISM FOR SCATTERING RATE}

Applying the Kinetic theory of gases to metals, Paul Drude ${ }^{14}$ formulated the formula for electrical conductivity:

$$
\sigma(\omega)=\frac{\omega_{p}^{2}}{4 \pi} \frac{1}{1 / \tau-i \omega},
$$

where $\omega_{p}^{2}=\frac{4 \pi n e^{2}}{m}$, is the plasma frequency, $n$ is the free carrier density, $m$ is the carrier mass and $1 / \tau$ is the scattering rate (the momentum relaxation rate). This formula can be derived from the momentum relaxation equation: $\frac{d \mathbf{p}}{d t}=\frac{-\mathbf{p}}{\tau}-e \mathbf{E}^{15}$. The Drude model is valid when electrons are treated as free and only impurity scattering is important (momentum relaxation coming from impurity scattering). But if electron-electron or electron-phonon scattering is important, this formula breaks down. With the developments in non-equilibrium statistical mechanics, especially by Kubo and Mori:146/17, it became possible to treat non-equilibrium problems and interactions (perturbatively!) and to arrive at the generalized Drude model (see Allen ${ }^{5}$, Holstein ${ }^{18}$, Gotze and Wolfe $\left.{ }^{\sqrt{19}}\right)$ :

$$
\sigma(\omega, T)=\frac{\omega_{p}^{2}}{4 \pi} \frac{1}{1 / \tau(\omega, T)-i \omega(1+\lambda(\omega, T))},
$$

where $\lambda(\omega, T)$ is the frequency dependent optical mass enhancement factor and $1 / \tau(\omega, T)$ is the frequency dependent scattering rate ${ }^{9}$. On comparing the real and imaginary parts, one can obtain the frequency dependent scattering rate as:

$$
\frac{1}{\tau(\omega, T)}=\frac{\omega_{p}^{2}}{4 \pi} \operatorname{Re}\left(\frac{1}{\sigma(\omega, T)}\right)
$$

and the mass enhancement factor $\lambda(\omega, T)$,

$$
1+\lambda(\omega, T)=-\frac{\omega_{p}^{2}}{4 \pi} \frac{1}{\omega} \operatorname{Im}\left(\frac{1}{\sigma(\omega, T)}\right) .
$$

The plasma frequency $\omega_{p}$ can be calculated using the sum rule analysis: $\int_{0}^{\infty} d \omega \sigma(\omega, T)=\frac{\omega_{p}^{2}}{8}$. From the experimental optical conductivity $\sigma(\omega, T), 1 / \tau(\omega, T)$ and $\lambda(\omega, T)$ can also be extracted.

Now, we would like to discuss the microscopic picture of $1 / \tau(\omega, T)$. Analytically, Allen $n^{5}$ has formulated the simple expression for $1 / \tau(\omega)$ for metals by taking the electron-phonon interaction into account. It relates the $1 / \tau(\omega)$ with electron-phonon spectral function $\alpha^{2} F(\omega)$ : 


$$
\frac{1}{\tau(\omega)}=\frac{2 \pi}{\omega} \int_{0}^{\infty} d \Omega(\omega-\Omega) \alpha^{2} F(\Omega)
$$

The above zero temperature formalism of Allen was generalized by Shulga et. al. ${ }^{7}$ for finite temperature:

$$
\begin{aligned}
\frac{1}{\tau(\omega, T)}= & \frac{\pi}{\omega} \int_{0}^{\infty} d \Omega I^{2} \chi(\Omega)\left[2 \omega \operatorname{coth}\left(\frac{\Omega}{2 T}\right)\right. \\
& -(\omega+\Omega) \operatorname{coth}\left(\frac{\omega+\Omega}{2 T}\right) \\
& \left.+(\omega-\Omega) \operatorname{coth}\left(\frac{\omega-\Omega}{2 T}\right)\right]
\end{aligned}
$$

But with an important change: the electron-phonon spectral function $\alpha^{2} F(\Omega)$ was replaced by electron-boson spectral function $I^{2} \chi(\Omega)^{9}$, to include the general case of scattering of electrons by boson degrees of freedom (other than phonons). Here the boson degrees of freedom could be bosons of electronic origin or the spin fluctuations ${ }^{20}$. With the limit $T \rightarrow 0$ in Eq. 6, one can obtain the Allen's result. Most importantly, the above formulae are not valid for the pseudogap phase of the cuprates. Because the above formulae assume the constant electronic density of states (EDOS) near the Fermi level. In cuprates, the electronic density of states modifies significantly at $T \leq T^{*}$-the signature of pseudogap- as has been confirmed by many other probes like ARPES, etc. Taking a non-constant electronic density of states into account, Mitrović and Fiorucci ${ }^{8}$ (in the context of metals) gave a relation for $T=0 K$ :

$$
\frac{1}{\tau(\omega)}=\frac{2 \pi}{\omega} \int_{0}^{\omega} d \Omega I^{2} \chi(\Omega) \int_{0}^{\omega-\Omega} d \omega^{\prime} \tilde{N}\left(\omega^{\prime}\right)
$$

where $\tilde{N}(\omega)=\frac{N(\omega)+N(-\omega)}{2 N(0)}$, is the normalized and symmetrized density of states ${ }^{8}$. To include the temperature effects in this picture, recently, Sharapov and Carbott $^{\sqrt{9}}$ has given an important general expression:

$$
\begin{aligned}
\frac{1}{\tau(\omega, T)}= & \frac{\pi}{\omega} \int_{0}^{\infty} d \Omega I^{2} \chi(\Omega) \int_{-\infty}^{\infty} d \omega^{\prime} \tilde{N}\left(\omega^{\prime}-\Omega\right) \\
& \times\left[n_{B}(\Omega)+1-f\left(\omega^{\prime}-\Omega\right)\right] \\
& \times\left[f\left(\omega^{\prime}-\omega\right)-f\left(\omega^{\prime}+\omega\right)\right],
\end{aligned}
$$

where $n_{B}(\omega)=\frac{1}{\exp (\beta \omega)-1}$ and $n_{F}(\omega)=\frac{1}{\exp (\beta \omega)+1}$ are the Boson and Fermi distribution functions and $\beta=$ $\frac{1}{k_{B} T}, k_{B}$ is the Boltzmann constant. Several models are discussed in literature for the form of the electronic density of states, for example, a step function, a flat density of states, a triangular form, etc ${ }^{9|21| 22}$. These toy model forms of $\tilde{N}(\omega)$ are modeled to take care of the formation of the pseudogap, but are quite far from the reality, for example, the pseudogap is known to evolve with doping

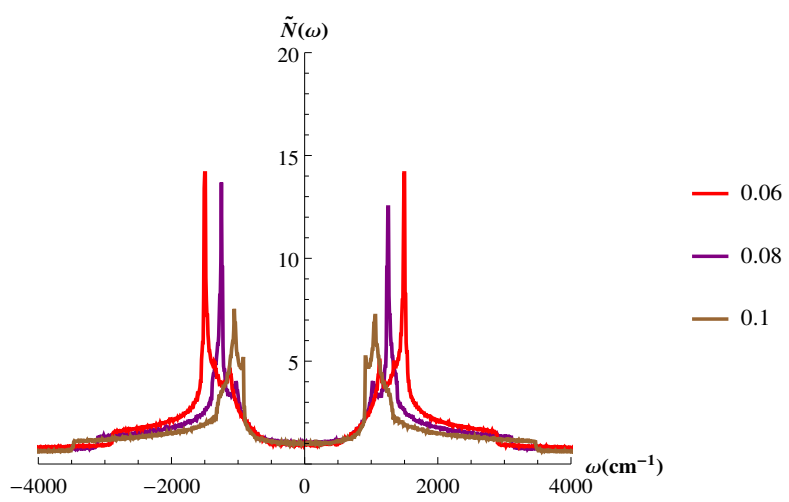

FIG. 2. The form of electronic density of states $\tilde{N}(\omega)$ using the YRZ model at different dopings such as $0.06,0.08$ and 0.10 . Here, the hopping parameter that have been used is $t_{0}=3600 \mathrm{~cm}^{-1}(0.45 \mathrm{eV})$. This plot shows the reduction of the magnitude of the pseudogap with the increase of the doping concentration.

but these forms do not have doping dependence. A realistic model for EDOS is the Yang-Rice-Zhang (YRZ) phenomenological model. The details of this model are given in Yang et. al.$^{12113}$ and are briefly presented in the appendix. The electronic density of states from YRZ model (see appendix) is given by

$$
N(\omega)=\sum_{\mathbf{k}} A_{Y R Z}(\mathbf{k}, \omega) .
$$

Here, $A_{Y R Z}(\mathbf{k}, \omega)=\frac{-1}{\pi} \operatorname{Im}\left[G_{Y R Z}(\mathbf{k}, \omega+i \epsilon)\right]$ is the YRZ spectral function. The form of $\operatorname{EDOS}(\tilde{N}(\omega))$ as calculated from YRZ model is shown in Fig. 2 at three different dopings $x=0.06,0.08$ and 0.1 . The Fermi surface corresponds to $\omega=0$. For $\omega<0$, the states are filled and for $\omega>0$, one has empty states. We notice from Fig. 2 that with decreasing doping, the gap (around $\omega=0$ ) widens. This is in accord with pseudogap crossover line in Fig. 1(a).

\section{NUMERICAL RESULTS AND ANALYSIS}

\section{A. Experimental features of ab-plane $1 / \tau(\omega, T)$ (qualitatively)}

Experimentally, the scattering rate of the cuprates has been studied by many authors such as Lee et. al. ${ }^{23}$, Timusk et. al. ${ }^{3]}$ and others ${ }^{20 \mid 24} 27$. A first important feature is the depression in the scattering rate at the low frequencies which is due to the opening of the pseudogap and occurs at $T<T^{* 23}$. This is shown schematically in Fig. 3(a), But no such type of depression has been seen at temperature $T>T^{*}$. Secondly, Timusk proposes two depressions picture ${ }^{3}$, one depression (at low frequency $\sim 600 \mathrm{~cm}^{-1}$ ) is due to the electron-boson interaction and the other (at high frequency $\sim 1000 \mathrm{~cm}^{-1}$ ) 


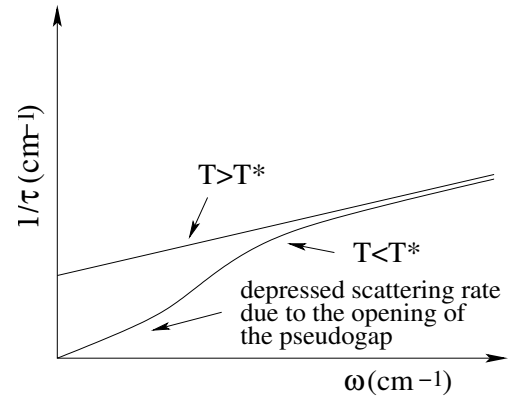

(a)

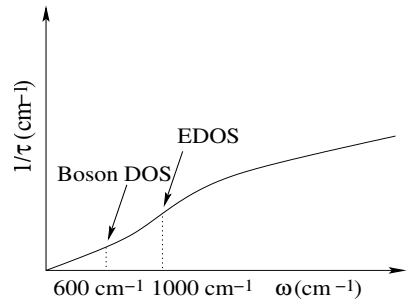

(b)

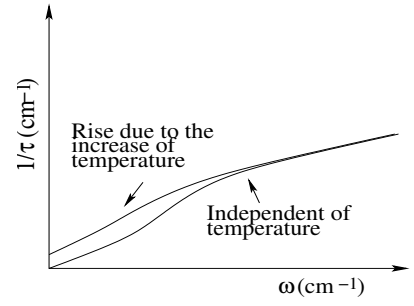

(c)
FIG. 3. The schematic diagrams showing the qualitative behaviour of the experimentally observed scattering rate. (a) shows the depression picture due to the pseudogap formation at $T<T^{*}$ and no such feature at $T>T^{*}$. (b) The two thresholds scenario, one around $600 \mathrm{~cm}^{-1}$ (possibly due to electron-boson scattering) and another around $1000 \mathrm{~cm}^{-1}$ (due to the opening of pseudogap in EDOS). (c) Dependence of $1 / \tau(\omega, T)$ on temperature at lower frequencies and its temperature independence at higher frequencies.

is due to the electronic DOS (refer Fig. 3(b)). A third and very interesting feature is the temperature evolution of the scattering rate (see Fig. 3(c) $)$. In this case, there is an increase in the $1 / \tau(\omega, T)$ with the increase of temperature only at low frequencies (roughly $\lesssim 1000 \mathrm{~cm}^{-1}$ ) and at high frequencies (roughly $\gtrsim 4000 \mathrm{~cm}^{-1}$ ), it does not show temperature dependence. In the next subsections, we analyze this observed behaviour of scattering rate using theoretical models.

\section{B. The Theoretical approach of Puchkov et. al. ${ }^{11}$}

Theoretically, Puchkov et al $\frac{11}{11}$ have investigated the behavior of the scattering rate at different temperatures. They consider the constant electronic density of states model (Allen's and Shulga-Dolgov-Maksimov's) and for the boson spectral function $\left(I^{2} \chi(\Omega)\right)$, they consider two models (1) the Lorentzian model $\Gamma \Omega /\left(\left(\Omega-\Omega_{E}\right)^{2}+(\Gamma)^{2}\right)$ with boson peak centered at $\Omega_{E}$ and width $\Gamma$, and (2) the single frequency $\Omega_{E}$ Einstein model $A \delta\left(\Omega-\Omega_{E}\right)$ with $A$ as electron-boson coupling constant. With the Lorentzian model for $I^{2} \chi(\Omega)$, the observed features are shown in Fig. 4(a). Here, we can see that at the low frequencies, there is a weak depression in the scattering rate at all temperatures. This depression comes from lower value

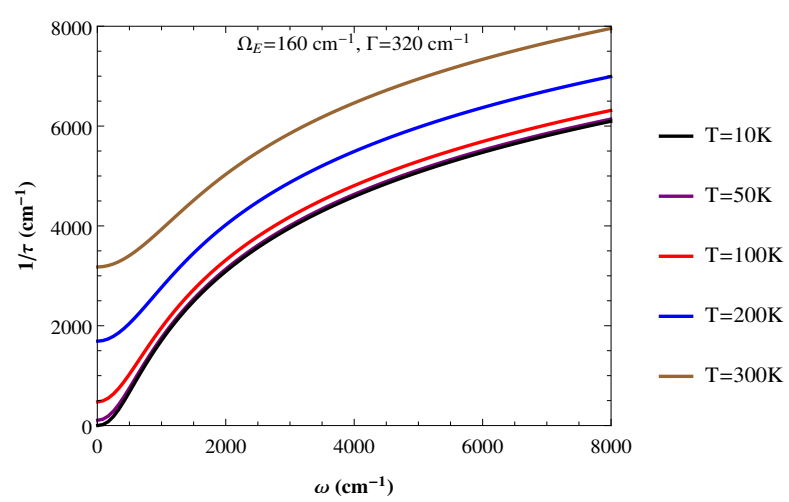

(a)

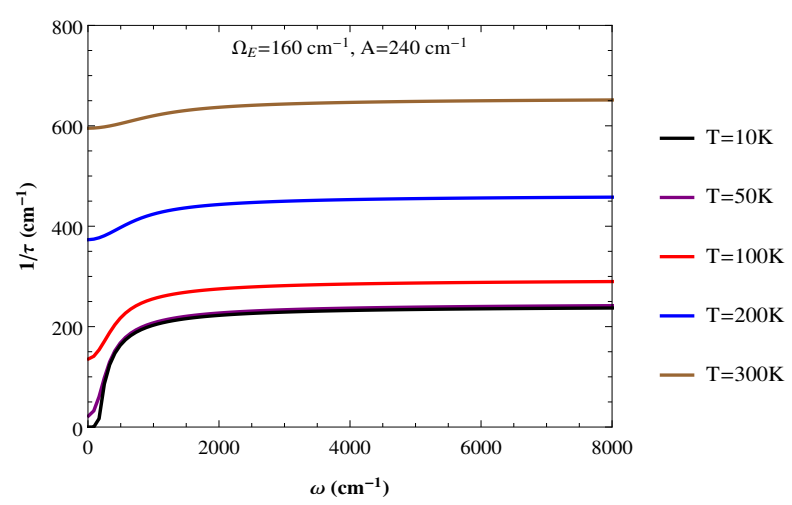

(b)

FIG. 4. The behaviour of the scattering rate with the variation in temperature using the approach given by Puchkov. (a) using the Lorentzian model, (b) with the Einstein model. Here, the parameters used are shown inset of the graph.

of boson density of states at frequencies less than $\Omega_{E}$ that causes less scattering of electrons (thus depression in the scattering rate). But in the experiment, the depression (at lower frequencies) appears only at $T<T * 23$. No depression occurs when $T>T^{*}$. This experimental fact cannot be resolved using the boson only model of Puchkov et. al ${ }^{11}$. It is hard to imagine that bosons suddenly becomes active at $T<T^{*}$ and inactive at $T>T^{*}$. We can also notice that with the increment in the temperature, the scattering rate increases at all frequencies in Puchkov et. al. approach whereas in experiment only the low frequency part is temperature dependent ${ }^{3}$ (refer Fig.3(c) ). In Fig. 4(b) with the Einstein model, the $1 / \tau(\omega, T)$ shows depression at lower frequencies and at lower temperature. But above about $1000 \mathrm{~cm}^{-1}$, it saturates over a wide frequency scale. Contrary to this, it varies linearly in the high frequency range in experiment. The basic reason for this contradiction seen in Puchkov et. al's work is due to the assumption of the constant EDOS at the Fermi surface. 


\section{Present approach: \\ Mitrović-Fiorucci/Sharapov-Carbotte scattering rate with YRZ electronic DOS}

To resolve these problems, we have performed numerical calculations for the scattering rate of cuprates using Mitrović-Fiorucci/Sharapov-Carbotte formalism with non-constant EDOS and we see good agreement with experiment. In these calculations, we have used the realistic YRZ mode $\frac{12 \mid 13}{13}$ for the EDOS. For the boson spectral function, we also consider two models (1) Lorentzian model $\Gamma \Omega /\left(\left(\Omega-\Omega_{E}\right)^{2}+(\Gamma)^{2}\right)$ and (2) Einstein model $A \delta\left(\Omega-\Omega_{E}\right)$ as used in the previous investigations 11 . With this approach, we have computed the $1 / \tau(\omega, T)$ in the underdoped region and at different temperature values. The used parameters are mentioned in the insets of the figures. But throughout the calculations, we have ignored the impurity contribution to the scattering rate (which contributes simple vertical constant shift of $1 / \tau(\omega, T))$.

As we mentioned before, a weak depression in Fig. 4(a) in Puchkov et. al.'s analysis comes only due to electronboson interaction and in the present approach (Fig. 5), the strong depression ${ }^{3 / 23}$ comes predominately from the opening of the pseudogap which is also observed in experiment (see Fig. 3 in Ref. 23). From Fig. 5, it is clear that there is a sharper depression in the scattering rate with the consideration of non-constant EDOS as compared to the constant EDOS. This strong depression feature clarifies the signature of the opening of the pseudogap, thus corroborates the experiment (refer Fig. 3 in Ref. 23). Another feature, the two thresholds picture as put forward by Timusk has also been noticed. First, around $160 \mathrm{~cm}^{-1}$, where the boson density of states is maximum, the quasi-particles start to scatter more and gives the signature of first threshold value. Further, in mid-infrared frequencies i.e. above $\sim 1200 \mathrm{~cm}^{-1}$, the scattering rate produces another threshold due to the contribution of the increased electronic density of states above the pseudogap. But at higher frequencies, i.e. above $3000 \mathrm{~cm}^{-1}$, due to the flattening of the EDOS (Fig. 2), the scattering rate flattens.

Now, we analyze the temperature dependence of the scattering rate at lower and higher frequency scale using both above mentioned models. First, using Einstein model, we can notice in the Fig. 6 that the scattering rate increases at all frequencies with the rise of temperature. But these results are not in accord with the experimental results. Because in this case, the boson mode has been set at a single frequency which is not an appropriate assumption. So, we have done the same calculations with the Lorentzian model. With this model, we can see in the Fig. 7(a) that there is temperature dependence in $1 / \tau(\omega, T)$ below a frequency $\sim 1000 \mathrm{~cm}^{-1}$. But $1 / \tau(\omega, T)$ is temperature independent at higher frequencies. These theoretically observed features are qualitatively in agreement with experiment. Thus we see that Sharapov-Carbotte scattering rate which include non -

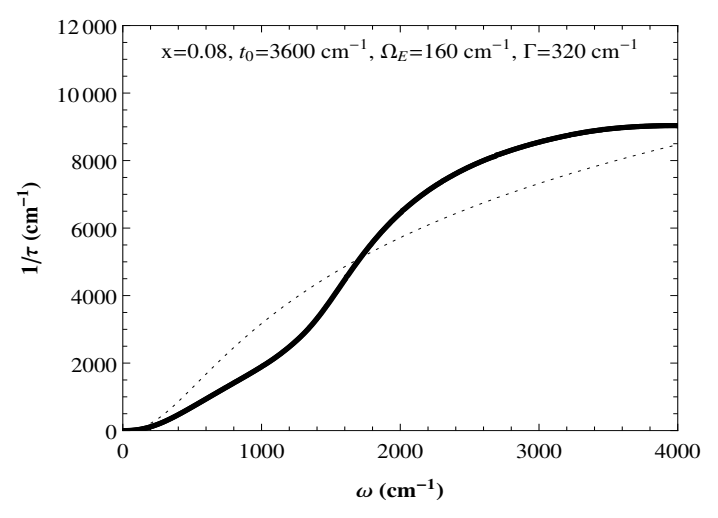

FIG. 5. Comparison plot for $1 / \tau(\omega, T)$ against the frequency with (solid line) and without (dotted line) the consideration of the electronic density of states. Here, the parameters used are set as $\Omega_{E}=160 \mathrm{~cm}^{-1}, \Gamma=320 \mathrm{~cm}^{-1}, \mathrm{x}=0.08$ and $t_{0}=3600$ $\mathrm{cm}^{-1}$.

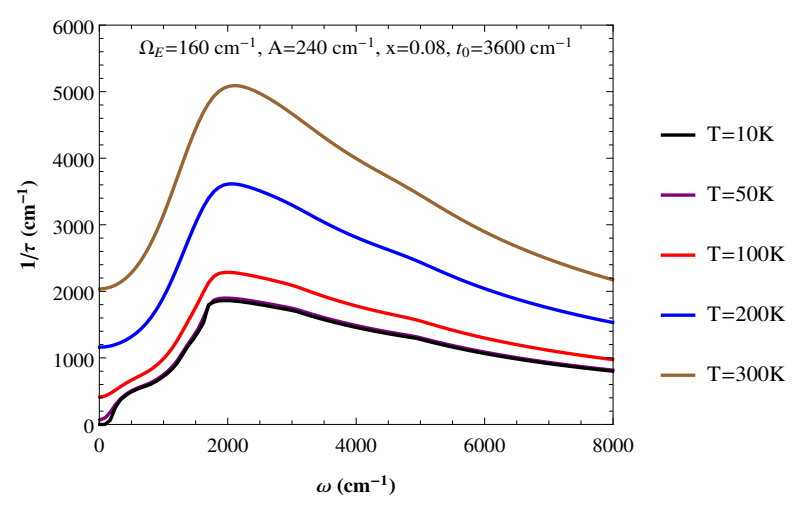

FIG. 6. With Einstein model, the temperature dependence character of the scattering rate at the doping concentration $x=0.08$. The scattering rate increases at all frequencies with the increase of the temperature.

constant EDOS is in accord with experiments.

\section{DISCUSSION AND CONCLUSION}

One of the important messages of the present investigation is that with constant EDOS (in Puchkov et. al. approach as shown in Fig 4(a) , it is not possible to account for the temperature independence of $1 / \tau(\omega, T)$ at higher frequency scale, whereas with non-constant EDOS (in Sharapov-Carbotte approach as shown in Fig.7(a), it is possible. This observation gives support to the Sharapov-Carbotte approach. The other points such as two thresholds picture and the strong depression formation in scattering rate has also been captured by the Sharapov-Carbotte formalism with the YRZ model as electronic density of states. The problems that come across with the Puchkov et. al's approach has been sorted 


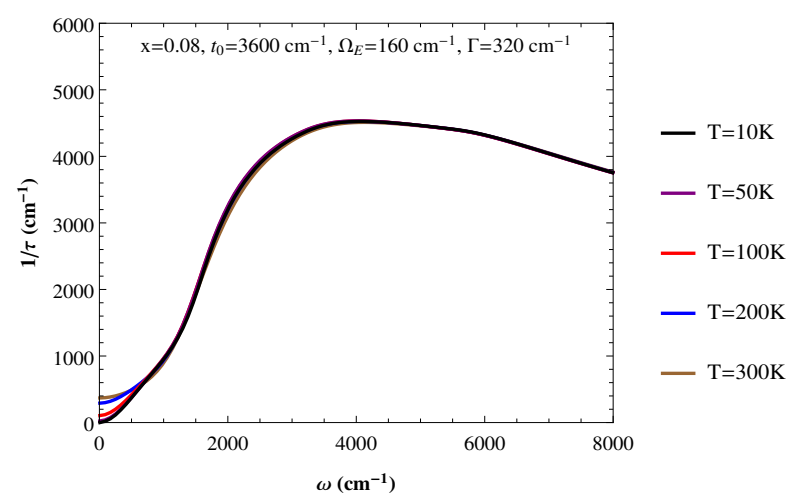

(a)

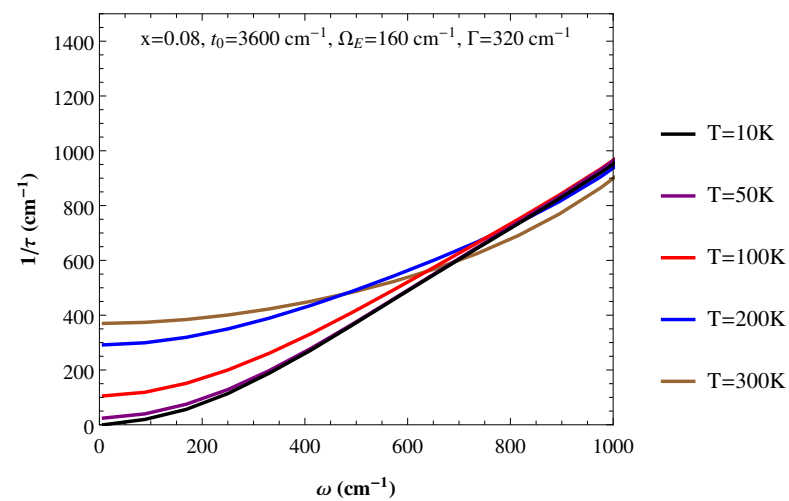

(b)

FIG. 7. (a) The behaviour of the scattering rate at the doping 0.08 with the change of the temperature. The scattering rate increases at the lower frequencies with the increase of the temperature and becomes temperature independent at higher frequencies. This is in accord with experiments. (b) The scattering rate on smaller frequency scale to see the clear view of the effect of temperature at lower frequency.

out. On comparing the results produced by Lorentzian and Einstein model, we have seen that the choice of Einstein model is inappropriate. Thus, the consideration of Lorentzian model for boson part and the YRZ model for electronic density of states in Sharapov-Carbotte scattering rate gives the qualitative picture of the experimental results.

But one important question remains, namely, in Fig $7(\mathrm{a})$ we notice that the scattering rate decreases with increasing frequency above about $4000 \mathrm{~cm}^{-1}$ whereas in experiment it increases linearly (refer Fig $3(\mathrm{~b})$ ) with frequency. The answer to this question lies in the fact that YRZ model is a low energy model and at higher $\omega, N(\omega) \propto 1 / \omega^{2}$ (from Eq. A4). This reduction in $N(\omega)$ at large $\omega$ (i.e. $\omega>>t_{0} \simeq 3600 \mathrm{~cm}^{-1}$ ) leads to reduced scattering rate (see Eq. 7). Another factor that causes the reduction in $1 / \tau(\omega, T)$ is the toy model choice i.e. the Lorentzian model for Boson density of states. In the Lorentzian model, Boson density of states peaks at $\omega=\Omega_{E}$ and decays as power law at large $\omega\left(\omega>>\Omega_{E}\right)$. This also cause the reduction in scattering rate at higher $\omega$. In real materials, Boson density of states is much more sophisticated than the simple Lorentzian mode ${ }^{28}$. However, at lower energy scale $\left(\omega \lesssim t_{0}\right)$ Sharapov-Carbotte formalism with Lorentzian model for Boson density of states qualitatively reproduce the experimental features seen in $1 / \tau(\omega, T)$.

\section{ACKNOWLEDGEMENT}

We are thankful to Prof. J. P. Carbotte for carefully reading the manuscript and suggesting many important corrections.

\section{Appendix A: Yang-Rice-Zhang ansatz}

The Yang, Rice and Zhang have proposed the single particle propagator:

$$
G(\mathbf{k}, \omega)=\frac{g_{t}(x)}{\omega-\xi(\mathbf{k})-\Sigma_{p g}(\mathbf{k}, \omega)},
$$

where the self energy term is $\Sigma_{p g}(\mathbf{k}, \omega)=\frac{\Delta_{p g}^{2}}{\omega+\xi_{0}(\mathbf{k})}$. Here $\omega$ is the frequency, $\mathbf{k}$ is the momentum, $\Delta_{p g}=$ $\frac{\Delta_{p g}^{0}}{2}\left(\cos \left(k_{x} a\right)-\cos \left(k_{y} a\right)\right)$ is the pseudogap and $\xi(\mathbf{k})$ is the band dispersion having hopping terms up to third nearest neighbor.

$$
\begin{gathered}
\xi(\mathbf{k})=-2 t\left(\cos \left(k_{x} a\right)+\cos \left(k_{y} a\right)\right)-4 t^{\prime} \cos \left(k_{x} a\right) \cos \left(k_{y} a\right) \\
\left.-2 t^{\prime \prime}\left(\cos \left(2 k_{x} a\right)-\cos \left(2 k_{y} a\right)\right)\right)-\mu_{p} . \\
\xi_{0}(\mathbf{k})=-2 t\left(\cos \left(k_{x} a\right)+\cos \left(k_{y} a\right)\right) .
\end{gathered}
$$

The used parameters are: $\mu_{p}$ is the shift in the chemical potential, $t, t^{\prime}, t^{\prime \prime}$, the hopping terms which are defined as $t=g_{t}(x) t_{0}+3 g_{s}(x) J \tilde{\chi} / 8, t^{\prime}=g_{t}(x) t_{0}^{\prime}$ and $t^{\prime \prime}=g_{t}(x) t_{0}^{\prime \prime}$. And $\Delta_{p g}^{0}=0.6 t_{0}\left(1-\frac{x}{0.2}\right)$. The Gutzwiller factors are $g_{t}(x)=\frac{2 x}{1+x}$ and $g_{s}(x)=\frac{4}{(1+x)^{2}}$. The values of other parameters are $J=t_{0} / 3, \chi=0.338, t_{0}^{\prime}=-0.3 t_{0}, t_{0}^{\prime \prime}=$ $0.2 t_{0}$ and $x$ is the doping concentration $12 \mid 13$.

The momentum averaged density of states in terms of spectral function ${ }^{9}$ is defined as:

$$
N(\omega)=\sum_{\mathbf{k}} A(\mathbf{k}, \omega)
$$

and $A(\mathbf{k}, \omega)=\frac{-1}{\pi} \operatorname{Im}\left[G_{Y R Z}(\mathbf{k}, \omega+i \epsilon)\right]$. Therefore, with the help of the equation A1, the density of states can be written as:

$$
N(\omega)=\frac{1}{\pi} \lim _{\epsilon \rightarrow 0} \sum_{\mathbf{k}} \frac{\epsilon g_{t}(x)\left(\omega+\xi_{0}(\mathbf{k})\right)}{\left(\left(\omega+\xi_{0}(\mathbf{k})\left(\omega-\xi(\mathbf{k})-\Delta_{p g}^{2}\right)^{2}+\epsilon^{2}\left(\omega+\xi_{0}(\mathbf{k})\right)^{2}\right.\right.}
$$


* pankaj@prl.res.in

1 J.G. Bednorz, and K.A. Müller, Z. Phys. B 64, (1986) 189.

2 P. W. Anderson, Science 235, (1987) 1196.

3 T. Timusk, Solid State Comm. 127, (2003) 337.

${ }^{4}$ H. Mori, Progr. Theoret. Phys. 34, (1965) 399.

5 P. B. Allen, Phys. Rev. B 3, (1971) 305.

${ }^{6}$ R. Kubo, Rep. Prog. Phys. 29, (1966) 255.

7 S.V. Shulga and O.V. Dolgov and E.G. Maksimov, Physica C 178, (1991) 266.

8 B. Mitrović and M. A Fiorucci., Phys. Rev. B 31, (1985) 2694.

9 S. G. Sharapov, and J. P. Carbotte, Phys. Rev. B 72, (2005) 134506.

10 Y. M. Dai, B. Xu, P. Cheng, H. Q. Luo, H. H. Wen, X. G. Qiu, R. P. S. M.Lobo, Phys. Rev. B 85, (2012) 092504.

11 A V Puchkov and D N Basov and T Timusk, J. Phys.: Condens. Matter 8, (1996) 10049.

12 K. Y. Yang,and T. M. Rice,and F. C. Zhang, Phys. Rev. B 73, (2006) 174501.

13 T. M. Rice, K. Y. Yang and F. C. Zhang, Rep. Prog. Phys. 75, (2012) 016502.

14 P. Drude, Annalen der Physik 308, (1900) 369.

15 N.W. Ashcroft, N.D. Mermin, Solid state physics, Science: Physics (Saunders College, 1976).

16 R. Kubo, J. Phys. Soc. Jpn. 12, (1957) 570.
17 H. Mori, Progr. Theoret. Phys. 33, (1965) 423.

18 T. Holstein, Ann. Phys. 29, (1964) 410.

19 W. Götze and P. Wölfle, Phys. Rev. B 6, (1972) 1226.

20 J. P. Carbotte and T. Timusk and J. Hwang, Rep. Prog. Phys. 74, (2011) 066501.

21 J Hwang and J Yang and J P Carbotte and T Timusk, J. Phys.: Condens. Matter 20, (2008) 295215.

22 J. Hwang, J Phys.: Condens. Matter 25, (2013) 295701.

${ }^{23}$ Y. S. Lee, K. Segawa, Z. Q. Li, W. J. Padilla, M. Dumm, S. V. Dordevic, C. C. Homes, Y. Ando, D. N. Basov,, Phys. Rev. B 72, (2005) 054529.

24 J. Hwang, J. Yang, T. Timusk, S. G. Sharapov, J. P. Carbotte, D. A. Bonn, R. Liang and W. N. Hardy, Phys. Rev. B 73, (2006) 014508.

${ }^{25}$ T. Timusk and B. Statt, Rep. Prog. Phys. 62, (1999) 61.

26 S. J. Moon, Y. S.Lee, A. A. Schafgans, A. V. Chubukov, S. Kasahara, T. Shibauchi, T. Terashima, Y. Matsuda, M. A. Tanatar, R. Prozorov, A. Thaler, P. C. Canfield, S. L. Bud'ko, A. S. Sefat, D. Mandrus, K. Segawa, Y. Ando and D. N. Basov, ArXiv e-prints, (2014) arXiv:1404.7233.

27 H. L. Liu, M. A. Quijada, A. M. Zibold, Y. D. Yoon, D. B. Tanner, G. Cao, J. E. Crow, H. Berger, G. Margaritondo, L. Forró , B. Hoan, J. T. Markert and M. Onellion, J. Phys.: Condens Matter 11 (1999) 239.

28 J. Hwang, J. P. Carbotte, Phys. Rev. B 89, (2014) 024502. 